\title{
Explicit learning of arbitrary and non-arbitrary action-effect relations in adults and 4-year-olds
}

\author{
Stephan A. Verschoor ${ }^{1}$, Rena M. Eenshuistra ${ }^{1}$, Jutta Kray ${ }^{2}$, Szilvia Biro ${ }^{1,3}$ and Bernhard Hommel ${ }^{1}$ * \\ ${ }^{1}$ Leiden University Institute for Psychological Research and Leiden Institute for Brain and Cognition, Leiden, Netherlands \\ ${ }^{2}$ Department of Psychology, Saarland University, Saarbruecken, Germany \\ ${ }^{3}$ Centre for Child and Family Studies, Leiden University, Leiden, Netherlands
}

Edited by:

David Sobel, Brown University, USA

Reviewed by:

Matthew Schlesinger, Southern

Illinois University, USA

Marissa Greif, Florida Atlantic

University, USA

${ }^{*}$ Correspondence:

Bernhard Hommel, Leiden University, Department of Psychology, Cognitive Psychology Unit, Wassenaarseweg 52, 2333 XZ Leiden, Netherlands. e-mail: hommel@fsw.leidenuniv.nl
Ideomotor theories claim that carrying out a movement that produces a perceivable effect creates a bidirectional association between the two, which can then be used by action control processes to retrieve the associated action by anticipating its outcome. Previous implicit-learning studies have shown that practice renders novel but action-contingent stimuli effective retrieval cues of the action they used to follow, suggesting that experiencing sequences of actions and effects creates bidirectional action-effect associations. We investigated whether action-effect associations are also acquired under explicit learning conditions and whether familiar action-effect relations (such as between a trumpet and a trumpet sound) are learned the same way as novel, arbitrary relations are. We also investigated whether these factors affect adults and 4-year-old children equally. Findings suggest that explicit learning produces the same bidirectional action-effect associations as implicitlearning does, that non-arbitrary relations improve performance without affecting learning per se, and that adults and young children show equivalent performance - apart from the common observation that children have greater difficulty to withstand stimulus-induced action tendencies.

Keywords: ideomotor theory, action-effect acquisition, 4-year-olds

\section{INTRODUCTION}

James' (1890) ideomotor theory claims that consciously thinking of an action goal automatically triggers the accompanying actions that will help to reach that goal. This ideomotor approach has proved to be very useful in interpreting and explaining goaldirected behavior. For example, if you intend to watch your favorite sitcom, it would be of good help if actions such as finding the remote control, switch on the TV, and looking for the right channel already get activated in order to accomplish your goal smoothly and correctly. If no actions get primed if an action plan comes to mind or if incorrect actions get triggered, such as getting a spoon or opening a door, reaching your goal would be severely disturbed or delayed. As the example shows, planning and control of goaldirected actions does not only require knowledge or expectations about the outcomes of these actions, it also implies that the relationship between the action and the action-outcomes has to be bidirectional in order to use action-outcomes as a trigger for action initiation: even though acquiring an action-effect relation implies that the cognitive representation of the given action gets activated before the representation of this action's effects (as the action precedes its effects), planning that action later on requires the representation of the effect to get activated before the representation of the action.

Ideomotor approaches to action planning and action control recently regained interest (Hommel, 1996; Elsner and Hommel, 2001; Hommel et al., 2001; Stock and Stock, 2004) and a number of studies have demonstrated that subjects indeed acquire and use bidirectional action-outcome contingencies to plan and guide their actions (for an overview, see Hommel and Elsner, 2009).
Most of these studies adopted the experimental design introduced by Hommel (1996; Elsner and Hommel, 2001), which comprises of two phases: an acquisition phase, in which an action-outcome contingency is learned, and a subsequent transfer phase, in which it is tested whether the acquired action-outcome contingency affects action control.

In an acquisition phase of this design subjects freely choose among several actions (say, the key-pressing response R1 or R2) and each action is consistently followed by an effect stimulus (say, sound E1 or E2). The effect stimulus is not relevant to the task and subjects are not encouraged in any way to attend to, or acquire the action-effect relationship. In the transfer phase the effect stimuli are now being presented as target stimuli, and the sound-key mapping can be either consistent $(\mathrm{E} 1 \rightarrow \mathrm{R} 1$ and $\mathrm{E} 2 \rightarrow \mathrm{R} 2)$ or inconsistent $(\mathrm{E} 1 \rightarrow \mathrm{R} 2$ and $\mathrm{E} 2 \rightarrow \mathrm{R} 1)$ with the keysound mapping of the acquisition phase. In general, it is found that subjects perform better during the transfer phase when an acquisition-consistent effect-action mapping is required as compared to an inconsistent mapping (e.g., Elsner and Hommel, 2001). Meaning that subjects respond faster and/or make fewer errors during the transfer phase when asked to respond to effects with the keys that previously caused these effects during acquisition, than when this mapping is reversed from that acquired during acquisition. This effect of mapping consistency on RTs and errors suggests that, despite the task-irrelevance of the action-effects and the action-effect relationship during acquisition, bidirectional action-outcome associations (R1 $\leftrightarrow \mathrm{E} 1$ and $\mathrm{R} 2 \leftrightarrow \mathrm{E} 2)$ were created during acquisition and this knowledge transfers to the transfer phase. Subjects with an acquisition-consistent mapping during 
the transfer phase apparently benefited from the action-outcome contingencies learned during the acquisition phase and use this knowledge to guide their actions in the transfer phase. It is also possible that performance on acquisition-inconsistent mappings was hampered by having learned inconsistent contingencies, but there is some evidence that positive transfer effects by far outweigh negative effects (Hommel, 2004).

The available studies on action-effect learning have used novel, arbitrary relations between action-effects, so that the amount of learning could be experimentally controlled. Moreover, as the novel action-effects are commonly task-irrelevant and subjects are often explicitly instructed to ignore them, the acquisition of action-effect associations must be considered implicit or at least non-intentional. Indeed, Elsner and Hommel (2004) were unable to find any relationship between the reported experience of a causal relationship between action and effect in the acquisition phase and the size of the consistency effect in the test phase. Verschoor et al. (submitted) recently showed that no awareness of action-effect relationships is required at all to nonetheless show action-effect learning in a transfer phase.

Even though it is theoretically important to demonstrate that spontaneous action-effect learning can take place under such "unfavorable" circumstances, one may argue that these circumstances are not particularly ecologically valid. Infants, children, and adult novices facing a new task will often actively explore the appropriateness and potential of alternative actions to reach a particular goal, and thus explicitly carry out specific actions to produce specific effects. In the present study, we investigated whether these circumstances also allow for the acquisition of bidirectional action-effect associations. Apart from ecological validity considerations, this research goal was motivated by some recent observations that seem to call into doubt that intentional action-effect learning leads to bidirectional associations.

As Verschoor et al. (submitted) demonstrated, requiring subjects to verbalize the causal relation between actions and effects eliminates the consistency effects in the transfer phase of the Elsner and Hommel (2001) paradigm. This might suggest that consciously representing causal relations emphasizes their unidirectional nature and thus either prevents the creation of bidirectional associations between action representations and effect representations or blocks them from impacting action control (Verschoor et al. submitted). A similar line of reasoning is suggested by more general views about associative and causal relations between events. While associative relations are supposed to be bidirectional and thus insensitive to the temporal sequence between the represented events, causal relations are usually seen as asymmetrical and irreversible (Hume, 1964; Hausman, 1998; see Waldmann, 1996). Consistent with Verschoor et al. (submitted), Arcediano et al. (2005) suggested that the use of verbal labels may stress the temporal relation between two events and as consequence could constrain the direction of the association. A study of Fenker et al. (2005) also assessed the difference between associative and causal relations. When subjects had to judge if sequences of two words (e.g., spark $\rightarrow$ fire or fire $\rightarrow$ spark) were presented in a causal (cause $\rightarrow$ effect) order or in a diagnostic order (effect $\rightarrow$ cause), they were much faster in judging the causal relations than the diagnostic relations. However when subjects were asked to judge if the same word sequences had an associative relation (spark $\leftrightarrow$ fire or fire $\leftrightarrow$ spark), the temporal order was of no importance anymore. It thus makes sense to assume that relations between actions and effects can be represented in both a bidirectional, associative manner that previous studies on implicit action-effect learning seem to have tapped, and a unidirectional, causal way that emphasizes, or is a consequence of emphasizing, the temporal sequence of actions and effects.

The question of this study thus was whether explicit actioneffect acquisition would be sufficient to eliminate bidirectional associations, which would put rather tight constraints on ideomotor theorizing in the context of action control, or whether explicit acquisition would be comparable to implicit acquisition as measured by many other studies on action-effect learning (e.g., Elsner and Hommel, 2001). In the acquisition phase of the present experiment we made up a cover story that explicitly related key-pressing actions to auditory effects in a causal fashion. The two response keys were introduced to symbolize (and labeled by pictures of) two sound-producing objects, a trumpet and a bell. Pressing the key was described as acting upon the respective object and subjects were instructed to make the musical instruments sound by pressing the corresponding keys. Consequently, subjects were made aware of the action, the outcome, and the sequential and causal relation between the two. If indeed, as Verschoor et al. (submitted) claim, explicit acquisition of action-effect knowledge is sufficient to render action-effect associations unidirectional, we would expect no effects of mapping on either RTs or errors.

In an additional attempt to consider more realistic situations, we were also interested to see whether pre-existing knowledge about the causal relationship between an action and its effects would alter the directionality of the respective association. To investigate that, we compared learning of a novel, unfamiliar, and arbitrary pairing of a key-pressing action with a tone, with learning of a pairing that we considered familiar to our subjects from pre-experimental experience (i.e., non-arbitrary, as defined in reference to that experience). To accomplish this contrast between non-arbitrary and arbitrary action-effects, we manipulated the actions effects so that one key would produce the sound that the respective object was known to produce (e.g., pressing the trumpet key produced a typical trumpet sound, from here on symbolized as $<$ toot $>$ ), whereas pressing the other key produced a novel and arbitrarily chosen sound (e.g., bell key $\rightarrow<$ piew $>$ ). There are at least two possible outcomes of this familiarity manipulation (a further possibility will be discussed later). First, one may assume that pre-existing associations facilitate bidirectional learning, so that the familiar relationship produces a stronger consistency effect. Second, however, it may be that knowing about the causal relationship between trumpets and the sound they produce strengthens the association from trumpet to $<$ toot $>$ but works against creating a bidirectional association. This should reduce or eliminate the consistency effects on RTs and error rates in the transfer phase for the non-arbitrary action-effects.

A final purpose of the present study was to compare the learning performance of adult subjects with 4 -year-old children. In previous studies we obtained evidence of action-effect acquisition in both adults and children, even though the performance profiles differed in detail (Eenshuistra et al., 2004; Kray et al., 2006): 
Whereas RTs showed equivalent mapping consistency effects in adults and children (with acquisition-consistent mappings yielding faster RTs), error rates exhibited considerably stronger consistency effects in children (with greater error rates in acquisitioninconsistent mappings). Indeed, in young children facing a justacquired action-effect seems to prime the associated action so strongly that they find it extremely difficult to impossible to select a different action if the instructions so demands - an observation that Eenshuistra et al. (2004) considered an example of goal neglect in the sense of Duncan (1995). Considering this systematic difference between adults and young children, we were interested to see whether comparable differences would be obtained under conditions that make the causal relation between actions and effects explicit, as in the present study, and whether possible effects of pre-experimental knowledge would affect adults and children in the same fashion.

\section{MATERIALS AND METHODS PARTICIPANTS}

Participants were 34 4-year-old children $(M=4.54, \mathrm{SD}=0.37)$ recruited from two kindergartens in the Netherlands and 35 undergraduate students from the Leiden University $(M=21.86$, $\mathrm{SD}=3.29)$. The data of two children and three students were excluded from the analysis because they were unable to perform the tasks and did not adhere to the task instructions, respectively. The children received small presents for participating, and the kindergartens received book tokens. Students received course credits or $€ 3.50$ for participating.

\section{TASKS AND STIMULI}

The experiment was divided into an acquisition phase and a transfer phase. Trials in the acquisition phase started with the presentation of a visual stimulus, a picture of Ernie or Bert from Sesame Street. A picture of Ernie signaled a go trial, which required a freely chosen left or right key press on a specially designed twokey keyboard. One key was marked with a picture of a trumpet and the other key with a picture of a bell. Pressing one of the two keys was always followed by a sound that was plausibly related to the picture marking the key (trumpet key followed by $<$ toot $>$ or bell key followed by $<$ tring $>$ ) resulting in a non-arbitrary action-tone relation. Pressing the other key led to the presentation of a sound that bore no pre-experimental relation to the picture label of the key (trumpet key and <piew $>$ or bell key and $<$ piew $>$ ) resulting in an arbitrary action-tone relation. For example, pressing the left trumpet key could be followed by a $<$ toot $>$ sound and pressing the right bell key by a <piew> sound. All key-sound mappings were balanced across subjects and all sounds were presented for $200 \mathrm{~ms}$. Ernie's picture remained on the screen until a response was given or until $7 \mathrm{~s}$ had passed. The next trial started $1500 \mathrm{~ms}$ after a response. When a picture of Bert was presented, no response was required (a no-go trial). The picture of Bert remained on the screen for $2 \mathrm{~s}$.

The transfer phase consisted of a go/no-go two-choice reactiontime task. Again a picture of Ernie signaled a go trial and a picture of Bert a no-go trial (no-go trials were used to make the task more appealing). The picture of Ernie staid on screen until a response was made or until $2 \mathrm{~s}$ had passed. In a no-go trial a picture of Bert without any sound was presented for $2 \mathrm{~s}$. The inter-trial interval was $1500 \mathrm{~ms}$.

A go trial started with the presentation of the picture of Ernie together with one of the two sounds that in the preceding acquisition phase served as action-effects $(200 \mathrm{~ms})$. The action-effects now served as imperative stimuli. Participants were to respond to the stimuli as quickly and accurately as possible according to a fixed stimulus-response (S-R) mapping. They were randomly assigned to either a consistent or an inconsistent $\mathrm{S}-\mathrm{R}$ mapping in the transfer phase. In the consistent mapping condition, the sound-key mapping matched the key-sound mapping of the acquisition phase (e.g., trumpet key $\rightarrow<$ toot $>$ and bell $\rightarrow<$ piew $>$ in the acquisition phase became $<$ toot $>\rightarrow$ trumpet key and $<$ piew $>\rightarrow$ bell key in the transfer phase). In the inconsistent mapping condition, the sound-key assignment was reversed (e.g., trumpet key $\rightarrow<$ toot $>$ and bell $\rightarrow<$ piew $>$ in the acquisition phase became $<$ piew $>\rightarrow$ trumpet key and $<$ toot $>\rightarrow$ bell key in the transfer phase). As in our previous study (Eenshuistra et al., 2004), each key press in the transfer phase triggered the same sound as in the acquisition phase, to avoid extinction of the action-effect associations. Previous research shows that this does not alter effect patterns on RT and errors in adults (Elsner and Hommel, 2001; Eenshuistra et al., 2004) but it greatly increases effect sizes in children. This finding was taken to suggest that action-effect knowledge in children quickly fades during transfer if the effect tones triggered by a keypress are absent.

Associatedness was coded with respect to the relation between the key label (trumpet or bell label) and the sound; e.g., responding with the trumpet key to <toot $>$ would fall into the non-arbitrary category while responding with the bell key to $<$ piew $>$ would count as arbitrary.

\section{PROCEDURE}

The acquisition phase consisted of 18 practice trials (12 go trials, 6 no-go trials) and 144 test trials (96 go trials and 48 no-go trials). Subjects were instructed that Ernie "likes music" and that they could "make music" for Ernie. They were told that they could choose freely which sound to make for Ernie by pressing the trumpet key or the bell key. The causal relation between pushing one of the keys and the resulting auditory effect was made explicit by instructing participants that "If you want the trumpet to make a sound you should push the trumpet key" and giving them the opportunity to explore the corresponding contingency by pressing the key. The same instruction followed for the bell key. Additionally, subjects were instructed that they could freely choose how often they pressed a key but that in total they had to press both keys about equally often. When participants persevered in pushing only one button during acquisition (predominantly children were prone to this), the experimenter reminded them to change keys now and then. Furthermore, they were told that Bert "does not like music" and that no response should be given when a picture of Bert appeared. To motivate subjects - especially the children - to complete the acquisition phase, they were told that Ernie was allowed to play until lunchtime. A picture of a clock that was colored for one third and two third was presented after 48 and 96 trials, respectively. After 144 trials the clock was completely colored, indicating that 
lunch time had reached and with that the end of the acquisition phase.

In the transfer phase participants were told that Ernie was making music and wanted them to participate. When a picture of Ernie appeared together with one of the two effect sounds, they should press the trumpet key and when they perceived Ernie together with the other effect sound they should press the bell key, thus motivating the (consistent or inconsistent) $\mathrm{S}-\mathrm{R}$ mapping required for the transfer phase. Again, subjects were instructed to withhold their response when Bert appeared, because "Bert hates music and likes the silence." As in the acquisition phase, subjects were informed about their progress in the transfer phase. However, now they were instructed that Ernie was allowed to make music until bedtime and the same clock procedure was used to indicate when bedtime had reached and the transfer phase had finished. The transfer phase consisted of 18 practice trials ( 12 go trials, 6 no-go trials) and 90 test trials ( 72 go trials and 18 no-go trials).

\section{RESULTS ACQUISITION PHASE}

Left and right hand responses were equally distributed [49.8 vs. $50.2 \%, t(63)=0.42, p>0.65]$ and equally fast $[F(1,60)=0.37$, $p>0.5]$. As expected, adults were substantially faster than 4 -yearolds (428 vs. $1215 \mathrm{~ms}$ respectively), $F(1,60)=290.09, p<0.001$, $\left.\eta_{\mathrm{p}}^{2}=0.83\right)$. Overall, responses were faster when they were followed by a sound effect that was pre-experimentally associated with the key label than by a sound that was not (805 vs. 839 ms respectively), $F(1,60)=6.82, p<0.02, \eta_{\mathrm{p}}^{2}=0.10$. This effect of associatedness interacted with age group $[F(1,60)=4.75$, $p<0.04, \eta_{\mathrm{p}}^{2}=0.07$ ] due to that the associatedness effect was reliable in the children ( 1185 vs. $1246 \mathrm{~ms}$ for non-arbitrary and arbitrary relations; $F(1,30)=5.92, p<0.05, \eta_{\mathrm{p}}^{2}=0.17$ ) but not in the adults $(p>0.20)$. No a priori effects of consistency were found.

\section{TRANSFER PHASE}

\section{RTs}

Mean RTs of the transfer phase were analyzed as a function of age group, associatedness (arbitrary versus non-arbitrary), and mapping (consistency versus inconsistent), with mean overall RT of the acquisition phase as covariate. Unsurprisingly, the adults were faster than the 4-year-old children (630 vs. $1120 \mathrm{~ms}$ for adults and children), $F(1,59)=47.41, p<0.001, \eta_{\mathrm{p}}^{2}=0.45$. As predicted by the hypothesis of bidirectional action-effect associations, the consistent mapping yielded faster responses than the inconsistent mapping (consistent mapping $801 \mathrm{~ms}$ inconsistent mapping $950 \mathrm{~ms}), F(1,59)=25.03, p<0.001, \eta_{\mathrm{p}}^{2}=0.30$ (see Figure 1). The interaction between age group and mapping consistency was not significant, $p>0.5$, suggesting that actioneffect learning was comparable in children and adults. Overall, responses were faster to non-arbitrary action-tone relations than to arbitrary relations ( 873 vs. $879 \mathrm{~ms}$ respectively), $F(1,59)=4.33$, $p<0.05, \eta_{\mathrm{p}}^{2}=0.07$. Like in the acquisition phase, this effect was numerically more pronounced in children (1096 vs. $1145 \mathrm{~ms}$ for non-arbitrary and arbitrary relations, respectively) than in adults, where it was reversed in sign ( 650 vs. $612 \mathrm{~ms}$ ), but the interaction between associatedness and age was not reliable, $p=0.19$. All other

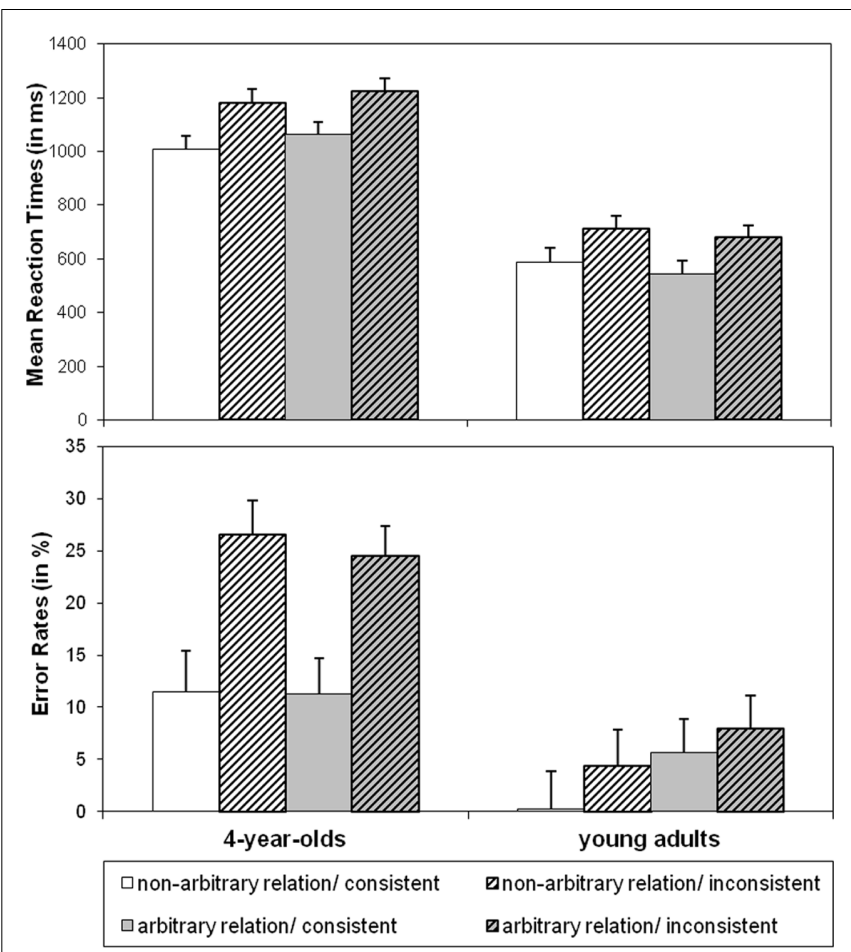

FIGURE 1 | Mean RTs (upper panel) and percentages of errors (lower panel) for performance on arbitrary and non-arbitrary sound-action relations as a function of age and consistency.

interactions with associatedness also failed to reach significance $(p>0.5)$.

\section{Accuracy}

Figure 1 shows the main effects for age, with adults making fewer errors than children [7 vs. $16 \% ; F(1,60)=18.92, p=<0.001$, $\left.\eta_{\mathrm{p}}^{2}=0.24\right]$. As predicted by our bidirectional association hypothesis, mapping yielded a main effect with fewer errors made with consistent mapping (consistent mapping 7\%, inconsistent mapping $16 \%), F(1,60)=17.87, p=<0.001, \eta_{\mathrm{p}}^{2}=0.23$. This effect was involved in a significant interaction with age group, $F(1$, $60)=7.38, p<0.01, \eta_{\mathrm{p}}^{2}=0.11$. Follow-up separate analysis for both age groups revealed that this was due to the mapping effect being significant in children $F(1,30)=15.34, p<0.001, \eta_{\mathrm{p}}^{2}=0.34$ (consistent mapping 8\%, inconsistent mapping 23\%) but not in adults, $p>0.11$, a pattern that replicates Eenshuistra et al. (2004). Overall, fewer errors were made with non-arbitrary action-tone relations than with arbitrary tone relations (10 vs. $12 \%$ for nonarbitrary and arbitrary relations, respectively), $F(1,60)=4.06$, $p<0.05, \eta_{\mathrm{p}}^{2}=0.06$, irrespective of mapping. All other interactions with associatedness failed to reach significance $(p>0.2)$. Moreover, children committed more no-go responses than young adults $(5.3$ vs. $0.5 \%), F(1,60)=6.51, p<0.02, \eta_{\mathrm{p}}^{2}=0.98$. This effect was not modulated by consistency $(p>0.25)$.

\section{DISCUSSION}

The aim of the present study was threefold. First, we were interested to see whether action-effect relations that are explicitly described 
as causal, and in a context where the effect is willfully produced by carrying out the respective action, are acquired in the same way as implicit action-effect relations. In particular, we asked whether explicit, intentional acquisition would lead to the creation of unidirectional associations between the representations of the action and the effect. If that would have been the case, no consistency effects would have been expected in the transfer phase. Given that such effects were obtained, and that the size of these effects is almost identical to what has been observed under implicit-learning conditions (Elsner and Hommel, 2001; Eenshuistra et al., 2004), it makes sense to assume that explicit learning does not differ from implicit-learning and that both types of learning result in bidirectional associations between action and effect representations. This is an important extension of previous findings and suggests that ideomotor logic applies to both the accidental pickup of possible future action goals during non-intentional movement (as typical for infants and young children) and the intentional acquisition during active exploration of action possibilities and affordances (as more typical for novices facing a new task and more experienced agents fine-tuning their actions). Finding evidence for bidirectional action-effect associations under explicit learning conditions provides further theoretical constraints on the explanation of Verschoor et al. (submitted) observation that asking subjects to repeat the task instructions in their own words prevents transfer from the acquisition to the transfer phase. Even though this manipulation is likely to render the causal relation between actions and effects in the acquisition phase explicit, the present findings do not suggest that this is sufficient to eliminate transfer effects in bidirectional binding.

The second aim of the present study was to compare the learning of novel, arbitrary relations between actions and effects, as often used in ideomotor studies, with the learning (or, better, strengthening) of action-effect relations that fit with preexperimental knowledge. Although the effects of pre-experimental knowledge on RT's and errors were small, they were coherent and reliable. Non-arbitrary, familiar relations affected performance and allowed for faster responding in both the acquisition and the transfer phase and more accurate responding in the transfer. However, there was no evidence that the type of relationship interacted with any other variable, including consistency. On the one hand, this means that our manipulation was successful in contrasting familiar and unfamiliar relations. On the other hand, however, the familiarity of a relation does not seem to modulate the format of the emerging or strengthened association. In particular, greater familiarity with the relationship between an action and its consequences does not seem to induce a unidirectional action $\rightarrow$ effect association per se. Given that our subjects were likely to know that trumpets and bells are causally related to the sounds they produce, we need to assume that they have corresponding unidirectional representations in principle but these representations were not activated in the context of the transfer phase. This confirms the consideration of Fenker et al. (2005) and Verschoor et al. (submitted), that unidirectional and bidirectional associations between events can coexist and be selectively activated in some contexts but not in others, presumably depending on instructions and task requirements. From a more general point of view, our findings are also consistent with the observation of Namy et al. (2004) that arbitrary relationships between events are learned just as well as non-arbitrary relationships. In their study, children were exposed to pairings between particular plastic toys and particular gestures, with some gestures bearing an iconic relationship to the toy (e.g., a hopping gesture and a rabbit) and others bearing an arbitrary relationship (a dropping motion and a rabbit). Learning was equally efficient in both conditions in 18-months-olds and 4-year-olds.

Our third aim was to compare transfer effects in young children and adults. Mapping consistency affected performance to the same degree in either group as far as reaction times are concerned. The error rates were also sensitive to consistency but more so in the 4-year-olds than in adults. This profile amounts to a perfect replication of previous observations (Eenshuistra et al., 2004; Kray et al., 2006). It suggests that young children were less able than young adults to keep the instructed S-R mapping active during the transfer phase, presumably due to less mature executive functioning and more resulting "goal neglect" (Eenshuistra et al., 2004). That is, stimuli related to possible action-effects seem to reactivate the associated action to such a degree that this tends to overwrite the S-R mapping held in working memory. However, in the trials where children do manage to keep the S-R mapping active and make a correct response, they show a similar effect of mapping on RTs as observed in the adults. In other words, the established task set seems to be equivalent in children and adults but the likelihood of keeping it sufficiently active is higher in adults. Most important for present purposes, however, this profile does not seem to change with explicit action-effect acquisition - as even the present effect magnitudes are comparable to implicit-learning conditions (Eenshuistra et al., 2004) - or the familiarity of the action-effect relation. Given that adults are likely to have had more experience with trumpets and bells and the sounds they produce than 4-yearold children have, this corroborates the conclusion, contrary to Verschoor et al. (submitted), that familiarity with the (unidirectional) causal connection between actions and effects does not prevent the creation of bidirectional associations between their representations.

To summarize, the present study shows that experiencing sequences of actions and effects induces the creation of bidirectional associations between action and effect representations, no matter what the degree of familiarity with related actioneffect contingencies and irrespective of whether the sequence is picked up implicitly or the action is explicitly carried out to produce the effect. This corroborates predictions from ideomotor approaches to action control (James, 1890; Hommel, 1996; Elsner and Hommel, 2001; Hommel et al., 2001) and demonstrates their broad applicability. At the same time, this converging evidence for bidirectional associations in children and adults raises the interesting issue how these associations, and their underlying learning processes, relate to causal learning proper, that is, to the acquisition of what Tolman and Brunswik (1935) have called "the causal structure of the environment." On the one hand, bidirectional associations can be considered to represent empirically derived knowledge about objective action-effect contingencies, which renders it some sort of causal knowledge. On the other hand, however, the bidirectionality of these associations - which the present findings seem to confirm - neglects the empirical and formal difference between cause and effect and, thus, violates 
the requirements of causal learning theories (e.g., Waldmann and Martignon, 1998; Gopnik et al., 2004). Given the observations that perceived causality does not depend on, and is no requirement for action-effect acquisition (Elsner and Hommel, 2004; Verschoor et al., submitted), this suggests that the acquisition of formal causal relationships is independent of ideomotor action-effect acquisition. This would imply that voluntary action control does not rely on a formal understanding of the world's causal structure.

\section{REFERENCES}

Arcediano, F., Escobar, M., and Miller, R. R. (2005). Bidirectional associations in humans and rats. J. Exp. Psychol. Anim. Behav. Process. 31, 301-318.

Duncan, J. (1995). "Attention, intelligence and the frontal lobes," in The Cognitive Neurosciences, ed. M. S. Gazzaniga (Cambridge, MA: MIT Press), 721-733.

Eenshuistra, R. M., Weidema, M. A., and Hommel, B. (2004). Development of the acquisition and control of action-effect associations. Acta Psychol. (Amst.) 115, 185-209.

Elsner, B., and Hommel, B. (2001). Effect anticipation and action control. J. Exp. Psychol. Hum. Percept. Perform. 27, 229-240.

Elsner, B., and Hommel, B. (2004). Contiguity and contingency in the acquisition of action effects. Psychol. Res. 68, 138-154.

Fenker, D., Waldmann, M. R., and Holyoak, K. J. (2005). Accessing causal relations in semantic memory. Mem. Cognit. 33, 1036-1046.

Gopnik, A., Glymour, C., Sobel, D. M., Schulz, L. E., and Kushnir, T. (2004). A theory of causal learning in children: causal maps and Bayes nets. Psychol. Rev. 111, 3-23.

Hausman, D. (1998). Causal Asymmetries. Cambridge: Cambridge University Press.

Hommel, B. (1996). The cognitive representation of action: automatic integration of perceived action effects. Psychol. Res. 59, 176-186.

Hommel, B. (2004). Coloring an action: intending to produce color events eliminates the Stroop effect. Psychol. Res. 68, 74-90.

Hommel, B., and Elsner, B. (2009). "Acquisition, representation, and control of action," in The psychology of action, eds E. Morsella, J. A. Bargh, and P. M. Gollwitzer (New York: Oxford University Press), 371-398.

Hommel, B., Müsseler, J., Aschersleben, their vicissitudes. Behav. Brain Sci. 24, 910-937.

Hume, D. (1964). Treatise of Human Nature, ed. L. A. Selby-Bigge, London: Oxford University Press. [Original work published 1739].

James, W. (1890). The Principles of Psychology. New York: Dover Publications. G., and Prinz, W. (2001). Codes and

\section{ACKNOWLEDGMENTS}

This research was funded by the Deutsche Forschungsgemeinschaft (SPP 1107) through grants to Jutta Kray (KR 1884/3-3) and Bernhard Hommel (HO 1430/8-3). Correspondence and requests for materials should be send to Bernhard Hommel, Leiden University, Department of Psychology, Cognitive Psychology Unit, Wassenaarseweg 52, 2333 AK Leiden, The Netherlands; hommel@fsw.leidenuniv.nl.

Kray, J., Eenshuistra, R., Kerstner, H. Weidema, M., and Hommel, B. (2006). Language and action control: the acquisition of action goals in early childhood. Psychol. Sci. 17, 737-741.

Namy, L. L., Campbell, A. L., and Tomasello, M. (2004). The changing role of iconicity in non-verbal symbol learning: a U-shaped trajectory in the acquisition of arbitrary gestures. J. Cogn. Dev. 5, 37-57.

Stock, A., and Stock, C. (2004). A short history of ideo-motor action. Psychol. Res. 68, 176-188.

Tolman, E. C., and Brunswik, E. (1935) The organism and the causal texture of the environment. Psychol. Rev. 42, 43-77.

Waldmann, M. R. (1996). "Knowledgebased causal induction," in Causa Learning: The Psychology of Learning and Motivation, Vol. 34, eds D. R. Shanks, K. J. Holyoak, and D. L. Medin (San Diego: Academic Press), 47-88.

Waldmann, M. R., and Martignon, L. (1998). "A Bayesian network model of causal learning," in Proceedings of the 20th Annual Conference of the Cognitive Science Society, eds M. A. Gernsbacher and S.
J. Deny (Mahwah, NJ: Erlbaum), 1102-1107.

Conflict of Interest Statement: The authors declare that the research was conducted in the absence of any commercial or financial relationships that could be construed as a potential conflict of interest.

Received: 08 May 2011; paper pend10 November 2011; published online: 27 February 2012.

Citation: Verschoor SA, Eenshuistra RM, Kray J, Biro S and Hommel B (2012) Explicit learning of arbitrary and nonarbitrary action-effect relations in adults and 4-year-olds. Front. Psychology 2:354. doi: 10.3389/fpsyg.2011.00354

This article was submitted to Frontiers in Developmental Psychology, a specialty of Frontiers in Psychology.

Copyright (C) 2012 Verschoor, Eenshuistra, Kray, Biro and Hommel. This is an open-access article distributed under the terms of the Creative Commons Attribution Non Commercial License, which permits use, distribution, and reproduction in other forums, provided the original authors and source are credited. ing published: 26 May 2011; accepted: 